\title{
BED SHEAR STRESS IN UNSTEADY FLOW
}

\author{
Paul A. Guard ${ }^{1}$, Peter Nielsen ${ }^{2}$ and Tom E. Baldock ${ }^{2}$
}

\begin{abstract}
Standard engineering methods of estimating bed shear stress using friction factors (i.e. $\tau_{b}=\frac{1}{2} f \rho u^{2}$ ) can fail spectacularly in unsteady hydrodynamic conditions. This paper demonstrates this fact using direct measurements of bed shear stresses under irregular waves using a shear plate apparatus. The measurements are explained in terms of the influence of the horizontal pressure gradient and the shear stresses acting on the surface of the plate. The horizontal fluid velocity at the edge of the boundary layer and the water surface elevation and slope were also measured. The paper demonstrates that the water surface measurements can be used to obtain accurate estimates of the forces on the bed, by employing Fourier analysis techniques or an innovative convolution integral method. The experimental results indicate that an offshore bed shear stress may be recorded while the free stream velocity remains onshore at all times. This demonstrates the failure of the standard engineering friction factor method in this scenario, since negative friction factors would be required. Important questions are raised regarding the appropriate definition for the bed shear stress when the vertical gradient of the shear stress is large. It is shown that it is problematic to define a single value for a "bed" shear stress in the presence of a strong horizontal pressure gradient. It is also argued that the natural driver for any model used to predict bed shear stress is the pressure gradient (or its proxy the free stream acceleration), rather than the velocity. This allows for accurate calculation of both acceleration effects (more rapid acceleration leads to a thinner boundary layer and higher shear stress) and also the direct action of the horizontal pressure gradient.
\end{abstract}

Keywords: bed shear stress, shear plate, convolution integrals, acceleration effects

\section{INTRODUCTION}

To illustrate the behaviour of the boundary shear stress in unsteady flow conditions, a series of experiments were carried out in the wave flume at the University of Queensland. The objective of the experiment was to measure the shear stress and pressure gradient forces on the bed of the flume under the passage of turbulent bores and unbroken wave trains. This study may be considered complementary to that of Liu et al (2007), which measured boundary layer characteristics under solitary waves. While previous studies have made use of similar shear plate apparatus (eg Riedel 1975, Mirfenderesk and Young, 2003, Babanin et al, 2005), there has been little investigation of the timing and phase differences between the bed shear stress and the near-bed velocity, which is the focus of this paper. The experimental setup is shown in Figure 1.

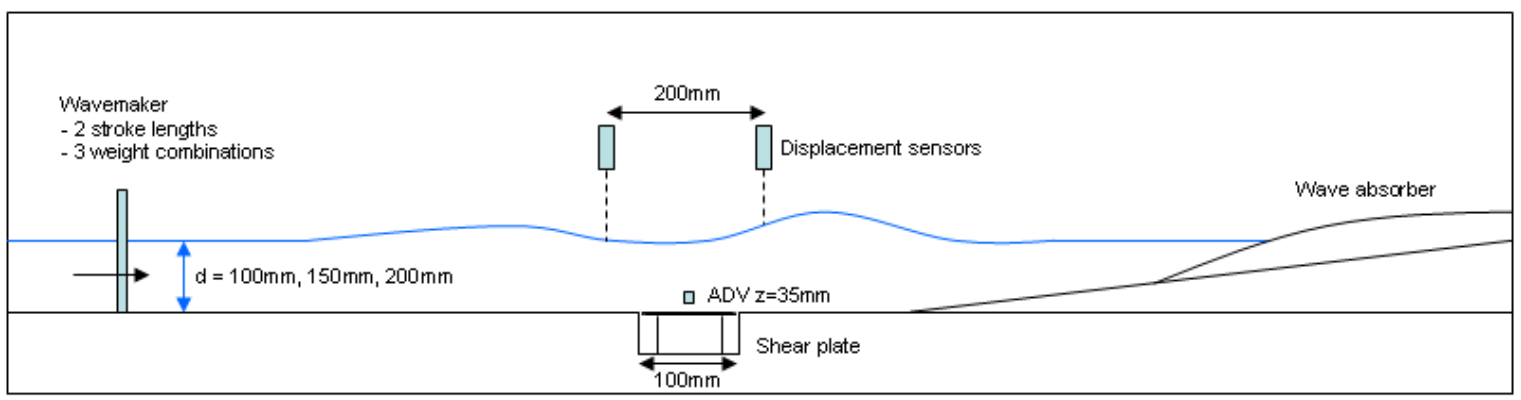

Figure 1 Experimental setup in the University of Queensland wave flume

\section{LABORATORY SETUP}

The starting condition for each experiment was quiescent water with a depth of $0.1 \mathrm{~m}, 0.15 \mathrm{~m}$ or $0.2 \mathrm{~m}$. A piston-type wavemaker was accelerated impusively using a system of falling weights. Three different weight combinations and two different stroke lengths produced surface profiles varying from strong bores to smooth unbroken wave trains. The near-bed free stream velocity directly above the shear plate was measured using an Acoustic Doppler Velocimeter (ADV) located 35mm above the bed. The water surface elevation and slope were measured by two Ultrasonic Displacement Sensors placed $200 \mathrm{~mm}$ apart, evenly spaced upstream and downstream of the shear plate. The horizontal distance

\footnotetext{
${ }^{1}$ BMT WBM, 490 Upper Edward St, Spring Hill, Qld, 4000, Australia. Email: paul.guard@bmtwbm.com.au

${ }^{2}$ School of Civil Engineering, University of Queensland, St Lucia, Qld, 4072, Australia
} 
between the wave paddle and the shear plate was approximately $15.2 \mathrm{~m}$, with a further $1.8 \mathrm{~m}$ between the shear plate and the wave absorber. The width of the flume was $0.86 \mathrm{~m}$.

The shear plate instrument is described fully in Barnes (2009), and a detailed diagram is shown in Figure 2. It comprises a $0.73 \mathrm{~mm}$ thick flat plate, dimensions $250 \mathrm{~mm}$ by $100 \mathrm{~mm}$, supported on smooth bearings and thin steel legs to allow horizontal movement in response to applied surface stresses and pressure forces. The horizontal displacement of the plate is measured by an eddy current sensor. The linear relationship between the plate displacement and the applied force is determined using a system of pulleys and weights as described in Barnes (2009). The natural frequency of the plate immersed in water was approximately $9 \mathrm{~Hz}$, well above the wave frequencies under investigation $(\sim 1 \mathrm{~Hz})$.

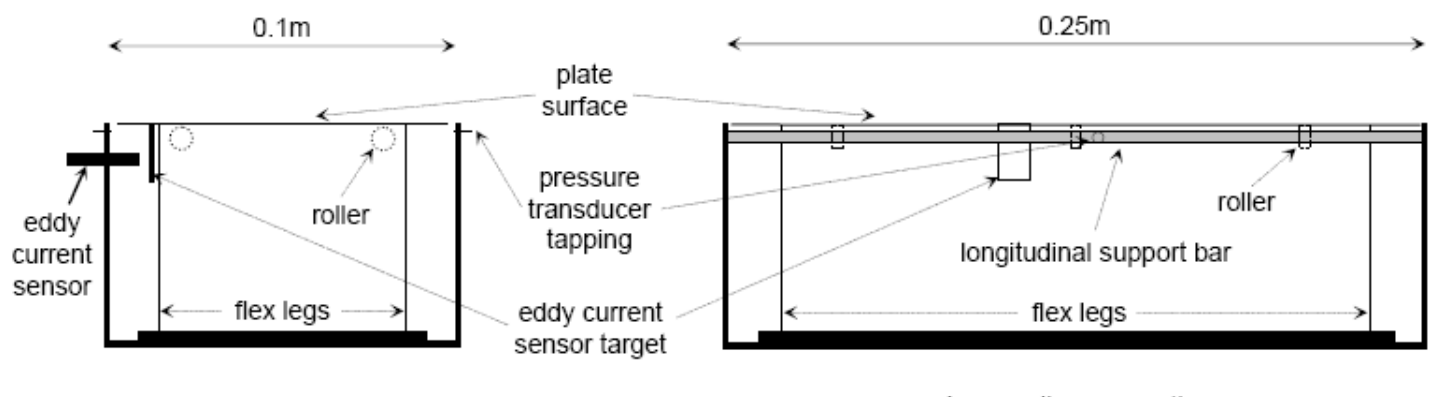

Cross-cell cross-section

Long-cell cross-section

Figure 2 Shear plate apparatus (from Barnes (2009))

The experimental conditions are summarised in Table 1. Each combination of pulling weights, stroke length and depth was repeated to demonstrate reproducibility of results, and only a representative case from each combination is presented here.

The type of wave produced by each weight combination is qualitatively described in Table 3, and included strong bores (single waves that break completely and then propagate as bores), medium bores (single waves that breaks partially and retain some of their original shape), weak bores (minor breaking, but wave shapes are retained) and unbroken waves (trains of unbroken waves where the first waves are much larger than those following).

The weight combinations used to accelerate the wavemaker paddle were $176 \mathrm{~N}$ (1), 227N (2) and 342N (3), where the code used in Table 1 is given in brackets. The short stroke length was approximately $1 \mathrm{~m}$, while the long stroke length was approximately $1.5 \mathrm{~m}$. The bottom surface of the flume and the top surface of the shear plate were covered in sandpaper with an equivalent Nikuradse roughness of $0.25 \mathrm{~mm}$.

\begin{tabular}{|l|l|l|l|l|}
\hline \multicolumn{2}{|l|}{ Table 1 Experimental conditions } \\
\hline Experiment no. & Depth $(\mathrm{mm})$ & Weight code & Stroke length & Wave type \\
\hline 014 & 100 & 1 & Long & Weak bore \\
\hline 015 & 100 & 2 & Long & Medium bore \\
\hline 016 & 100 & 3 & Long & Strong bore \\
\hline 021 & 100 & 1 & Short & Unbroken waves \\
\hline 023 & 100 & 2 & Short & Weak bore \\
\hline 024 & 100 & 3 & Short & Medium bore \\
\hline 030 & 150 & 1 & Long & Unbroken waves \\
\hline 032 & 150 & 2 & Long & Weak bore \\
\hline 035 & 150 & 3 & Long & Medium bore \\
\hline 039 & 150 & 1 & Short & Unbroken waves \\
\hline 041 & 150 & 2 & Short & Weak bore \\
\hline 043 & 150 & 3 & Short & Medium bore \\
\hline 045 & 200 & 1 & Long & Unbroken waves \\
\hline 046 & 200 & 2 & Long & Unbroken waves \\
\hline 050 & 200 & 3 & Long & Unbroken waves \\
\hline 053 & 200 & 1 & Short & Unbroken waves \\
\hline 055 & 200 & 2 & Short & Unbroken waves \\
\hline 057 & 200 & 3 & Short & Unbroken waves \\
\hline
\end{tabular}




\section{BED SHEAR STRESS AND NEAR BED VELOCITY CALCULATIONS}

\section{Fourier techniques}

When evaluating the forces on a sediment particle on the bed we must consider both the shear stress at the surface of a roughness element and pressure gradient forces.

If the boundary layer is laminar and the flow is horizontally uniform, the applied shear stress $\tau_{b}$ on the top surface of the plate is given by

$$
\mathrm{T}(\omega)=\rho \sqrt{i v \omega} U_{\infty}(\omega)
$$

where $\mathrm{T}(\omega)$ and $U_{\infty}(\omega)$ are the Fourier transforms of $\sqrt{\tau_{b}(t)}$ and $u_{\infty}(t), \omega$ is angular frequency, $v$ is kinematic viscosity, $\rho$ is fluid density and $i$ is the imaginary unit. Thus if we can calculate or measure $u_{\infty}(t)$ (the velocity just outside the boundary layer) we can calculate the laminar bed shear stress. We obtain the Fourier transform of $u_{\infty}(t)$, multiply by the frequency response function $\rho \sqrt{i v \omega}$, and obtain the inverse Fourier transform of the result.

Apart from this skin friction force there is also a pressure gradient force applied to the shear plate. This is a body force applied to the entire volume of the plate, so the equivalent force per unit area of the plate is given by

$$
\tau_{\text {equiv }}=\frac{\partial p}{\partial x} \frac{\text { Volume }_{\text {plate }}}{\text { Area }_{\text {plate }}}=\frac{\partial p}{\partial x} t_{\text {plate }}
$$

where $t_{\text {plate }}$ is plate thickness and $p$ is pressure at the bed. If the pressure is hydrostatic, this equation simplifies to

$$
\tau_{\text {equiv }}=\rho g \frac{\partial \eta}{\partial x} t_{\text {plate }}
$$

where $\eta$ is the measured surface elevation and $g$ is gravitational acceleration.

However, in the laboratory experiment described here the surface curvature was frequently large and therefore the pressure distribution deviated substantially from hydrostatic. We make use of the well known expression for pressure variation under sine waves (see eg Dean and Dalrymple (1991)) and obtain

$$
P(\omega)=\rho g\left(h+\frac{\mathrm{H}(\omega)}{\cosh (k h)}\right)
$$

where $P(\omega)$ and $\mathrm{H}(\omega)$ are the Fourier transforms of the bottom pressure $p(t)$ and surface displacement $\eta(t)$ respectively, and $h$ is water depth.

The wave number $k$ is found using an explicit approximation to the linear dispersion relation (Fenton and McKee, 1990)

$$
k=\frac{\omega^{2}}{g}\left(\operatorname{coth}\left(\frac{\omega^{2} h}{g}\right)^{3 / 4}\right)^{2 / 3}
$$

We can also estimate the near bed velocity using the recorded surface elevation timeseries. The appropriate frequency domain transfer function is

$$
U_{\infty}(\omega)=\frac{\omega}{\sinh (k h)} \mathrm{H}(\omega)
$$

Note for comparison the shallow water (hydrostatic pressure) expression

$$
u_{\infty}(t)=\sqrt{\frac{g}{h}} \eta(t)
$$

By comparing the calculated $u_{\infty}(t)$ with the measurements of $u_{\infty}(t)$ from the ADV it can be seen that the Fourier analysis approach using equation (6) yields a much better estimate than (7), see Figure 3. The deviation of the measurements from the calculations after $t=10.5 \mathrm{~s}$ is due to the return flow which develops once the waves reach the beach. 


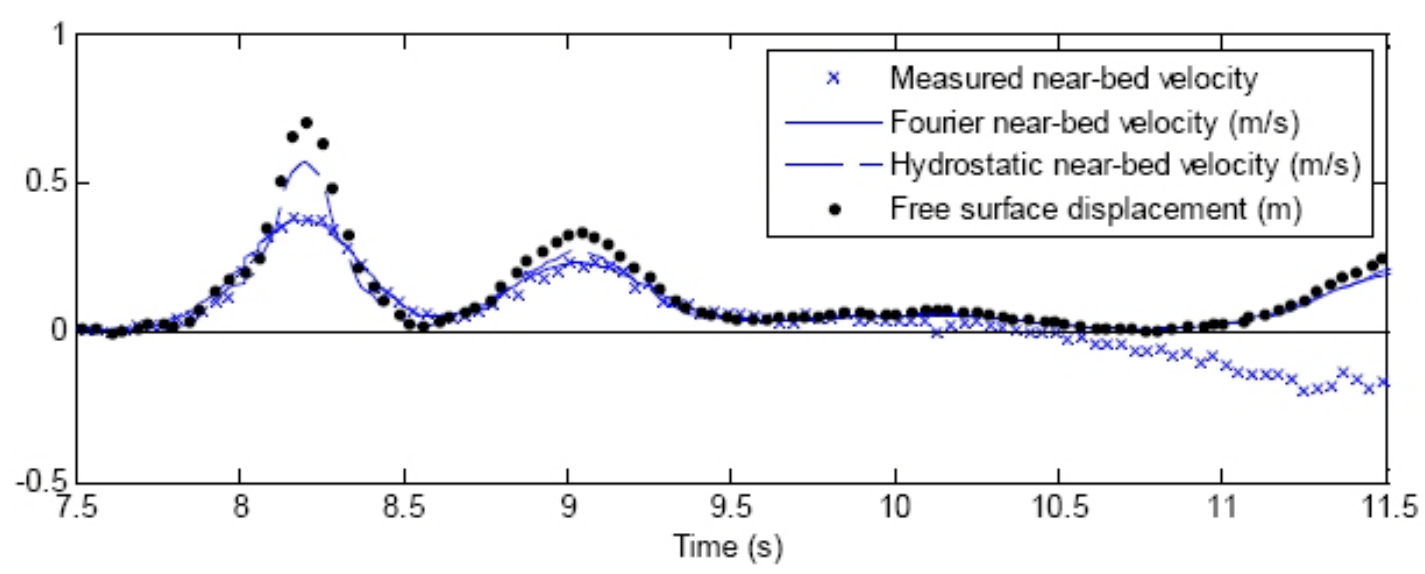

Figure 3 The near-bed velocity, $u_{\infty}(t)$, calculated from measured $\eta(t)$ using Fourier techniques eqn (6) and the hydrostatic equivalent eqn (7), compared to the $u_{\infty}(t)$ measured by the ADV.

The near-bed velocity (eqn (6)), laminar shear stress (eqn (1)) and equivalent stress due to the pressure gradient (eqns (2) and (4)) were calculated for all of the test cases in Table 1. Figure 4 shows a comparison of these calculations with the measured data for test cases 016, 041 and 057. These three cases represent typical results for a strong bore (016), weak bore (041) and unbroken wave (057).

\section{Convolution integral calculations}

The forces on the bed can also be estimated without the use of Fourier techniques. Multiplication in the frequency domain is equivalent to convolution in the time domain. The appropriate convolution integral calculation corresponding to (1) is

$$
\tau(t)=\rho \sqrt{\frac{v}{\pi}} \int_{0}^{t} \frac{\partial u_{\infty} / \partial t^{\prime}}{\sqrt{t-t^{\prime}}} d t^{\prime}
$$

This calculation was performed using the experimental data for $u_{\infty}(t)$, with the same result as the calculation from (1). A discrete form of the integral is used and a special technique must be adopted where $t-t^{\prime}$ goes to zero. Integrating the over the discrete interval from $t-\Delta t / 2$ to $t$, we find

$$
\int_{t-\frac{\Delta t}{2}}^{t} \frac{1}{\sqrt{t-t^{\prime}}} d t^{\prime}=\sqrt{2} \Delta t
$$

Therefore the value of $\partial u_{\infty} / \partial t$ corresponding to this initial timestep in the convolution integral is multiplied by the coefficient $\sqrt{2} \Delta t$. Torsvik and Liu (2007) described techniques for efficient calculation of convolution integrals such as (9).

A similar convolution integral technique may be used to estimate the bottom pressure instead of the frequency domain calculation in equation (4). The appropriate impulse response function is the inverse Fourier transform of the frequency response function $1 / \cosh (k h)$, i.e.

$$
f(x)=\frac{1}{2 \pi} \int_{-\infty}^{\infty} \frac{1}{\cosh k h} e^{i k x} d k=\frac{1}{2 h \cosh \frac{\pi x}{2 h}}
$$

This impulse response function is shown in Figure 5. 
a)

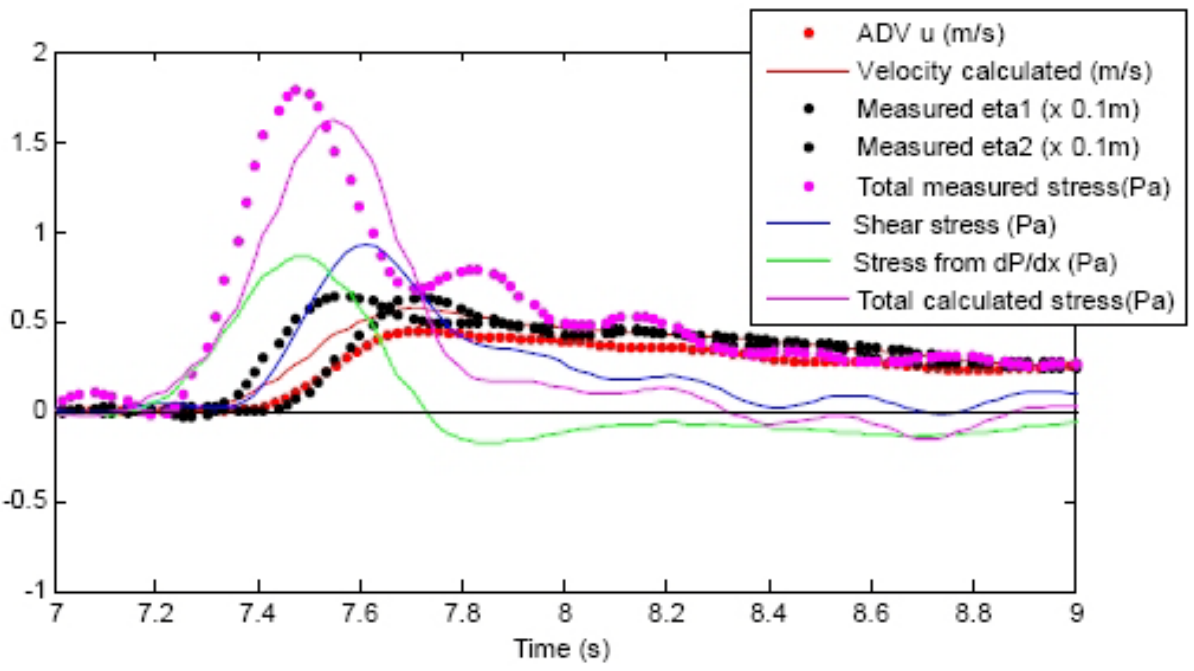

b)

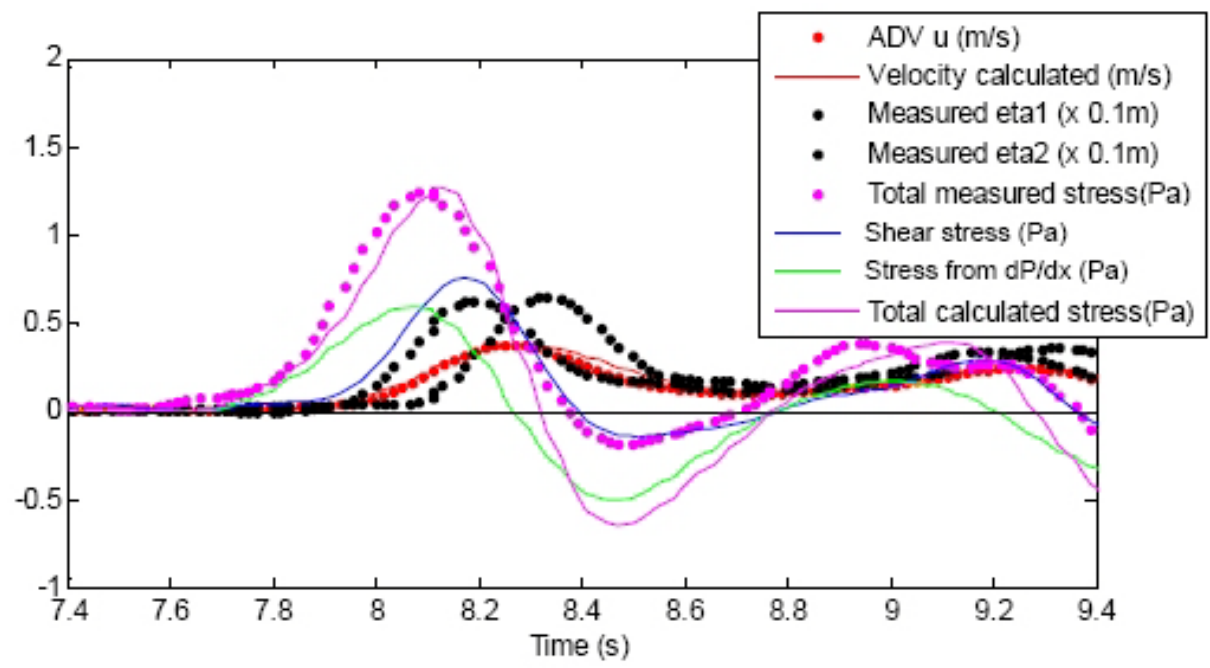

c)

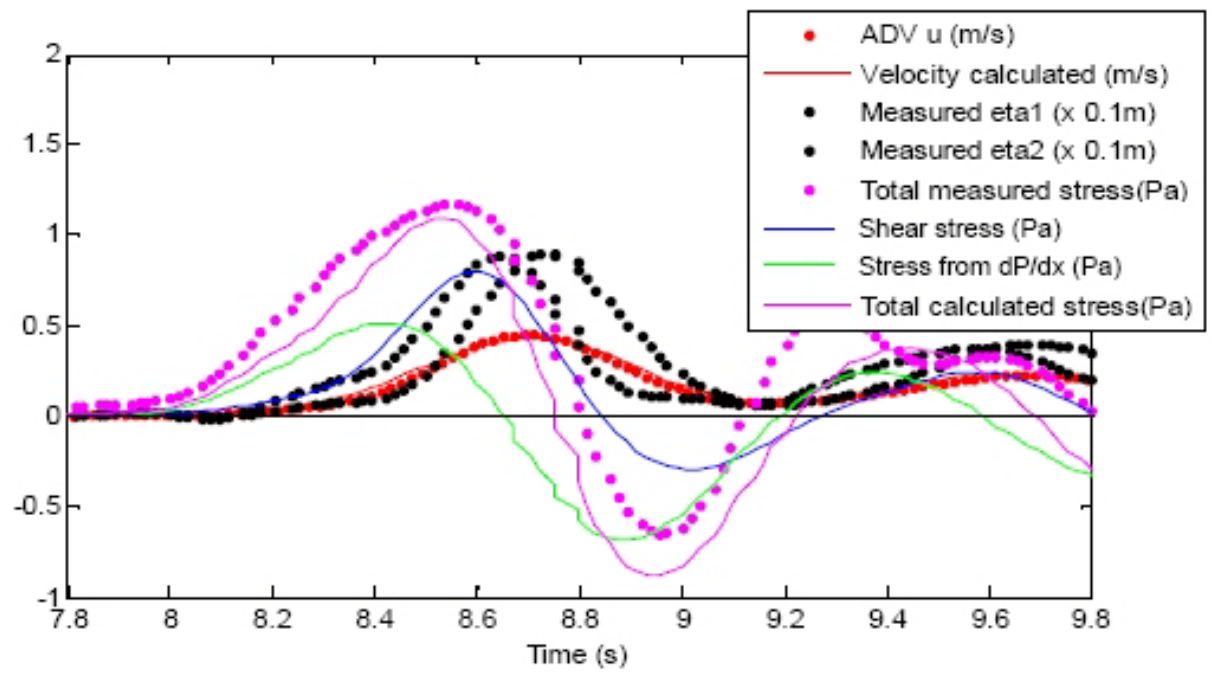

Figure 4. Measured and calculated near bed velocity and total stress on the shear plate, for experiment numbers a) 016, b) 041 and c) 057. The total stress is the sum of the laminar shear stress and the equivalent stress due to the horizontal pressure gradient. 


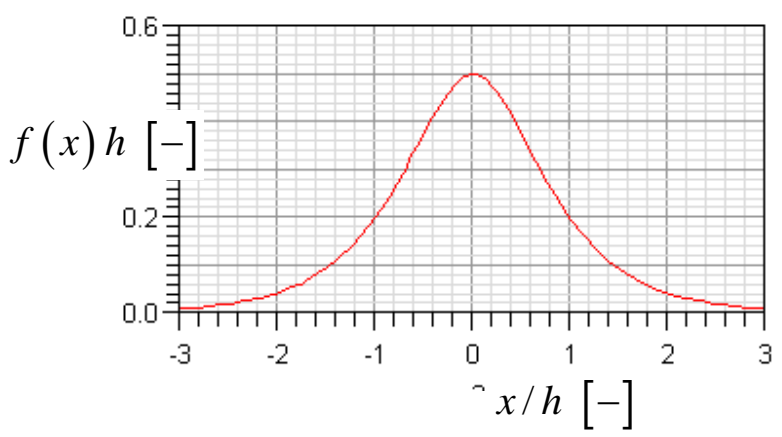

Figure 5 Impulse response function for bottom pressure from surface elevation

Note that the magnitude of the impulse response function is very small at distances of more than three water depths away from $x=0$. This allows us to truncate the convolution integral, which would otherwise be infinite in extent. Hence the appropriate $x$-domain calculation for $p(t)$ is

$$
p(t)=\rho g\left(h+\int_{-3 h}^{3 h} \frac{\eta(x)}{2 h \cosh \frac{\pi\left(x-x^{\prime}\right)}{2 h}} d x\right)
$$

This involves integration in the spatial domain $x$, but we have measured the surface elevation $\eta(t)$ as a function of time only. To make the transformation from $\eta(t)$ to $\eta(x)$ we assume that the waves travel with constant form over the interval of integration, and that therefore

$$
d x=\sqrt{g h} d t
$$

The results from equations (8) and (11) are compared to the results from equations (1) and (4) in Figure 6. The laminar shear stress calculation (eqns (1) and (8)) are in closer agreement than the pressure calculation (eqns (4) and (11)), probably because of the approximation (12) in the pressure calculation.

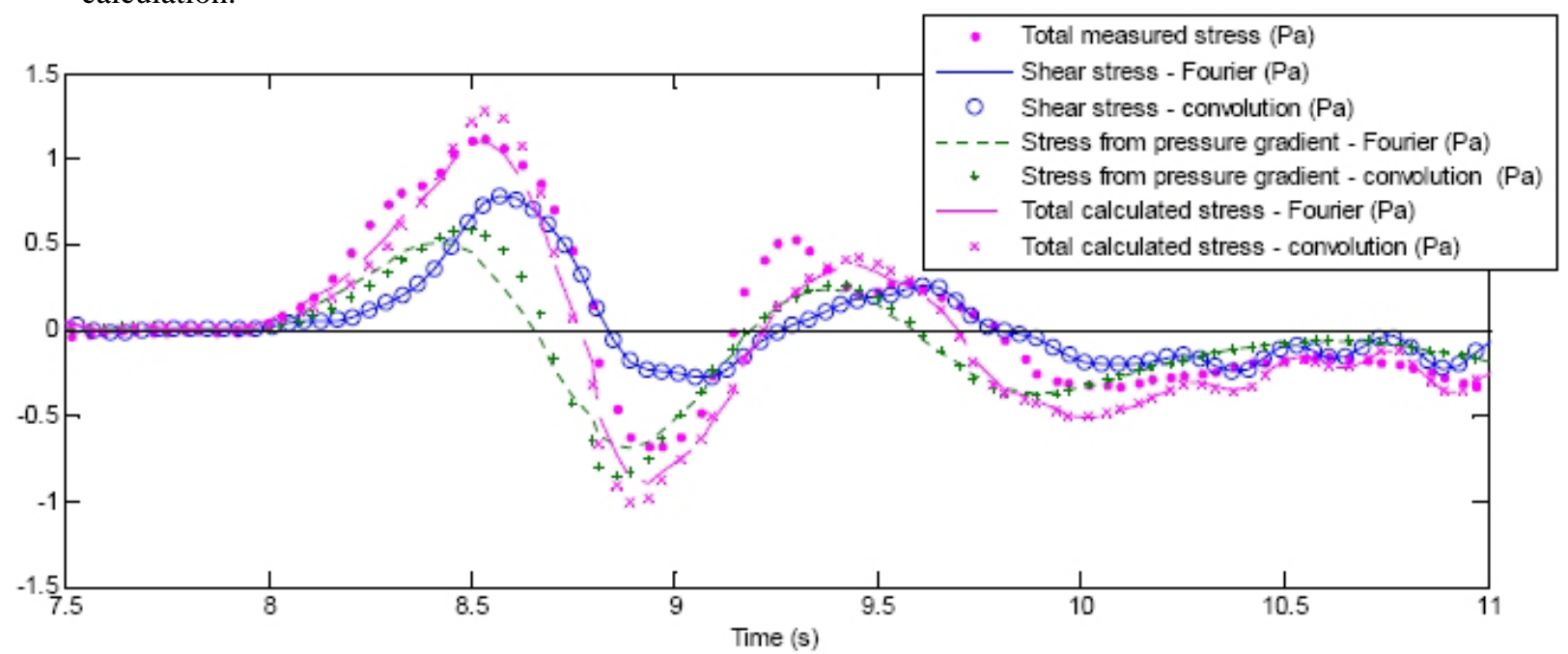

Figure 6. A comparison of calculations using Fourier analysis (equations (1) and (4)) and convolution integrals (equations (8) and (11))

\section{DISCUSSION OF RESULTS}

The results presented in Figure 4 show that there is substantial agreement between the calculations and measurements for the near-bed velocity and shear plate total stress. Note in particular that for the laboratory test cases considered, the bed shear stress (eqn (1)) and the equivalent bed shear stress due to the pressure gradient (eqn (2)) are of approximately equal magnitude.

We can demonstrate that the boundary layer in these experiments is indeed laminar. An estimate of the wave Reynolds number for each of the test cases can be made by first calculating an approximate water particle semi-excursion length $A$ : 


$$
A=\int_{0}^{t_{r}} u_{\infty}(t) d t
$$

where $t_{r}$ is the time at flow reversal. Then the Reynolds number is given by

$$
\frac{A u_{\infty \max }}{v}= \begin{cases}3.38 \times 10^{5} & \text { for case } 016 \\ 1.27 \times 10^{5} & \text { for case } 041 \\ 1.60 \times 10^{5} & \text { for case 057 }\end{cases}
$$

and $A / k_{s}$ was of the order $1 \times 10^{3}$, so by comparison with wave boundary layer experimental data (Riedel 1975) the experimental conditions were likely within the laminar or transitional regime. The close agreement between calculation and measurement justifies the assumption of a laminar surface shear stress.

Another important observation from Figure 4 is that although the near-bed velocity $u_{\infty}(t)$ remains positive during the passage of the leading waves, there is a reversal of the stress on the plate surface in cases 041 and 057. This is the same observation made by Liu et al (2007) from their Particle Image Velocimetry (PIV) measurements, which indicated a flow reversal in the boundary layer but not in the free stream. Note that traditional friction factor formulae of the form $\tau_{b}=\frac{1}{2} \rho f u^{2}$ will fail in this scenario, since negative friction factors would be required in order to get the correct bed shear stress. Liu et al (2007) also found that the solution for the full nonlinear boundary equation including advective terms did not differ substantially from the linearised result (eqn (1)).

One surprising observation is that the shear plate begins to measure a force due to the pressure gradient up to 0.2 seconds before the ultrasonic displacement sensors measure the arrival of the wave. This indicates that a bore travelling into still water will start to influence sediment particles on the bed before the bore reaches them. Of course, because of the substantial thickness of the shear plate the pressure gradient force we have measured is relatively large. Since the plate thickness is $0.73 \mathrm{~mm}$, it is equivalent to us choosing a level $0.73 \mathrm{~mm}$ into the bed at which to evaluate the shear stress. In a mobile bed the level of the initial point of failure of the sediment matrix will depend on the degree of bed compaction and specific gravity of the sediment. (see, for example, Sleath (1999)).

It is important to note that whenever the flow is unsteady, ie whenever there are large horizontal pressure gradients leading to high rates of fluid acceleration, there is an equivalently large vertical gradient in the near-bed shear stress. This is seen in the calculation for the shear stress at elevation $z$ :

$$
\tau(z, t)=\rho \int_{z}^{\infty} \frac{\partial}{\partial t}\left[u_{\infty}(t)-u(z, t)\right] d z^{\prime}
$$

This shear stress vanishes outside the boundary layer, and if the integral is continued down into a non-moving bed of sediment the shear stress gradient reaches a maximum of $\partial \tau / \partial z=\partial p / \partial x$. This makes it difficult to define a single value for the "bed" shear stress in the presence of a horizontal pressure gradient, since the shear stresses experienced by sediment particles at different elevations vary substantially.

The driver for both the frequency domain and time domain calculations presented here is the free stream acceleration. This is the logical choice for any boundary layer model input, since it is a proxy for the horizontal pressure gradient which is the physical driver of the boundary layer dynamics. Models which use the free stream acceleration as their main input are able to accommodate acceleration effects (more rapid acceleration leads to a thinner boundary layer and higher shear stress) and the direct action of horizontal pressure gradients automatically.

The present study has illustrated some of the important characteristics of bed shear stress in unsteady flow. Further experiments should be carried out over a wider range of both laminar and turbulent conditions. 


\section{ACKNOWLEDGMENTS}

The first author would like to acknowledge the financial support of BMT WBM and the University of Queensland.

\section{REFERENCES}

Babanin, A.V., Young, I.R., and Mirfenderesk, H., 2005. Field and laboratory measurements of wave-bottom interaction. Proceedings of the 17th Australasian Coastal and Ocean Engineering Conference, 20-23 September 2005, Adelaide, pp. 293-298

Barnes, M.P., 2009. Measurement and modelling of swash zone bed shear stresses. University of Queensland, PhD thesis. 157 pp.

Dean, R.G. and Dalrymple, R.A., 1991. Water wave mechanics for engineers and scientists. Advanced series on ocean engineering, 2. World Scientific, Singapore.

Fenton, J.D. and McKee, W.D., 1990. On calculating the lengths of water waves. Coastal Engineering, 14(6): 499-513.

Liu, P.L.-F., Park, Y.S.P. and Cowen, E.A., 2007. Boundary layer flow and bed shear stress under a solitary wave. J Fluid Mech, 574: 449-463.

Mirfenderesk, H. and I.R. Young, (2003) Direct measurements of the bottom friction factor beneath surface gravity waves, International Journal of Applied Ocean Research. Vol. 25, 269-287.

Riedel, H.P., 1972. Direct Measurement of Bed Shear Under Waves. Queen's University, PhD Thesis, 109pp.

Sleath, J.F.A., 1999. Conditions for plug formation in oscillatory flow. Continental Shelf Research, 19: 1643-1664.

Torsvik, T. and Liu, P.L.F., 2007. An efficient method for the numerical calculation of viscous effects on transient long waves. Coastal Engineering, 54(3): 263-269. 